\title{
Reliable Delivery of Event Data from Sensors to Actuators in Pervasive Computing Environments ${ }^{\star}$
}

\author{
Sudip Chakraborty, Nayot Poolsappasit, and Indrajit Ray \\ Computer Science Department \\ Colorado State University \\ Fort Collins, CO 80523, USA \\ \{sudip, nayot, indrajit\}@es.colostate.edu
}

\begin{abstract}
The event-condition-action (ECA) paradigm holds enormous potential in pervasive computing environments. However, the problem of reliable delivery of event data, generated by low capability sensor devices, to more capable processing points and vice versa, needs to be addressed for the success of the ECA paradigm in this environment. The problem becomes interesting because strong cryptographic techniques for achieving integrity impose unacceptable overhead in many pervasive computing environments. We address this problem by sending the data over the path from the sensor node to the processing point that provides the best opportunity of reliable delivery among competing paths. This allows using much weaker cryptographic techniques for achieving security. The problem is modeled as a problem of determining the most reliable path - similar to routing problems in networks. We propose a trust-based metric for measuring reliability of paths. The higher the trust value of a path the more reliable it is considered. We propose techniques for estimating the trust levels of paths and propose a new algorithm for identifying the desired path.
\end{abstract}

\section{Introduction}

Pervasive computing technology has the potential to impact numerous applications that benefit society. Examples of such applications are emergency response, automated monitoring of health data for assisted living, environmental disaster mitigation and supply chain management. Pervasive computing uses numerous, casually accessible, often invisible, computing and sensor devices. These devices are frequently mobile and/or embedded in an environment that is mobile. Most of the time they are inter-connected with each other, with wireless or wired technology. Being embedded in the environment and interconnected allow pervasive computing devices to exploit knowledge about the operating environment in a net-centric manner. This enables pervasive computing applications to provide a rich new set of services and functionalities that are not otherwise possible through conventional means. Pervasive computing applications frequently rely

\footnotetext{
* This work was partially supported by the U.S. Air Force Research Laboratory (AFRL) and the Federal Aviation Administration (FAA) under contract F30602-03-1-0101 and by the U.S. Air Force Office of Scientific Research under contract FA9550-07-1-0042. Any opinions, findings, and conclusions expressed in this publication are solely those of authors and do not necessarily represent those of the AFRL, the FAA, or the AFOSR.
} 


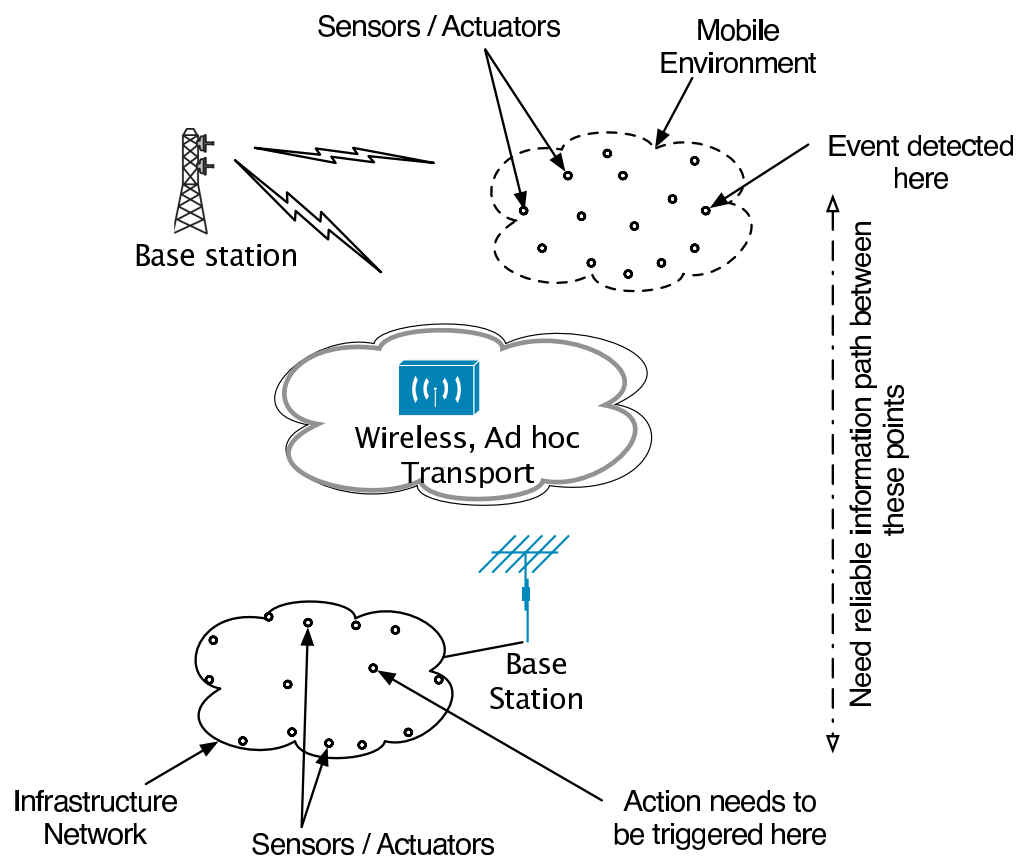

Fig. 1. Pervasive computing environment involving remote event detection and action triggering

on event-triggered obligation policies to operate in a dynamic environment. An obligation policy is associated with events, conditions, subjects, objects and actions. When the event of interest occurs and the associated conditions evaluate to true, the subjects perform the specified actions on the objects. Events are typically identified and captured by embedded sensing devices and actions are actuated by similar embedded devices. Processing of captured event data for evaluation of conditions are, on the other hand, mostly performed at remote processing nodes or base stations. This is because the sensing and actuating devices embedded in the environment are frequently of very low performance capabilities including low computing, low storage and low power. Thus a major challenge in a pervasive computing environment is to provide a path for propagating sensor data to processing nodes and action data to actuating nodes. Reliability of the paths is important. The data should be delivered with the minimum possible error and in as timely a manner as possible.

The reliable transmission path requirement imposes significant challenges in pervasive computing environments. A pervasive computing application can seldom assume a reliable network infrastructure for communication. In a conventional setting, a node that generates a message forwards it to a neighboring reliable node. The receiving node in turn forwards the message to another fixed node that is known a priori. This procedure is followed till the message reaches the destination. Every node in this process knows at least one other reliable node in the path towards the destination to which the message 
can be handed over. Frequently a node will know about more than one other node and thus have a choice of a better node. The nodes are static, that is they do not change their location and consequently the links between the nodes are stable. This and the proper use of strong cryptographic techniques, easily facilitate reliable delivery of messages in conventional settings. In a pervasive computing environments, on the other hand, mobility of nodes (sensing, processing or actuating) is frequently considered an asset. Figure 1 depicts the scope of the problem. Nodes are not locationally stable; instead they continuously change their coordinates. Thus, a node that needs a message delivered cannot rely on another fixed node to forward the message but has to make use of one or more nodes that happen to be within reachable distance at that particular moment. In addition, since a majority of these nodes are low capability devices (in the sense of low computational capabilities, low storage and low power provisions), use of strong cryptographic techniques needs to be ruled out. Moreover, in hostile environments these nodes get easily compromised. Under such circumstances it will enormously benefit a pervasive computing application if the path that provides most opportunity of reliable delivery of messages is presented to it. Determining an appropriate path within a network is the problem of routing. In this paper we revisit this problem in the context of pervasive computing environments.

The problem of routing in mobile ad hoc networks have been addressed before [1|2|3|4|5|6|8|9|10|11]. Among these [16/7|10] study cryptographic techniques for securing the routing protocol. Some use public key cryptography to encrypt the endto-end transmission of routing messages. Others use digital signature techniques to authenticate routing messages at the peer-to-peer level connection. However, these cryptographic techniques incur high computation and storage overhead which limit their use in sensor devices. Use of secret key techniques instead of public keys alleviate this problem to some extent although at the expense of added complexity. Moreover, key distribution and management is a big problem in secret key based systems. It is difficult to establish a key distribution or certification authority in mobile ad hoc environments. Ensuring the availability of a key distribution center or a certifying authority is almost impossible given the unstable nature of the network.

The Hermes protocol developed by Zouridaki et al. [11] proposes using trustworthiness of its neighbors for routing. The trust values are computed under the assumption that they follow the beta probability distribution. The parameters of the beta distribution come from the empirical observation of the forwarding behavior. Thus, nodes that maintain a good and steady forwarding history have more trust and confidence on them. The route is established for the most trusted path. However, the major problem of this work is its complete reliance on forwarding history for measuring trust. A malicious node can easily fake this history thus presenting itself as a trusted node. Other similar works include [12 13 14]. Among these [13] proposes a signal stability-based adaptive routing (SSR) where the routes are selected based on signal strength. This work looks promising; however it does not discuss how to measure this signal strength quantitatively. In [12] the authors propose an on demand secure routing protocol where the metric is based on past history. Yi et al. [14] present a security-aware ad hoc routing protocol (SAR) in which a route is selected on the basis of degree of 'security guarantee' that the route provides. If two routes have same guarantee then the shorter path is chosen. 
The security metric can be specified by standard security properties like timeliness, ordering, authenticity etc. However, the paper does not discuss how we can measure these properties quantitatively.

We propose a trust-based routing protocol for pervasive computing environments. Our protocol determines the most reliable path under currently determinable properties of the system to forward a packet from source to destination. A node in the pervasive computing environment is any entity that is able to forward a packet. It can be a sensor node, a mobile device like a PDA or a cellular phone, a powerful computing device or even an actuator like a switch. Reliability of a node is measured in terms of a trust value for the node. Each node determines its neighbor's trust based on physical properties of the neighbor that can be directly observed, the neighboring node's behavior history (i.e., results of past interactions) and recommendation (or rating) about the neighbor from other neighbors. The resulting trust value is used to generate the 'cost of forwarding', or simply cost. The cost metric is inversely related to the trust metric, that is, higher the trust (reliability) on the node, lower is the cost. This cost is associated with links in the network. We next modify the widely used distance vector routing protocol using these costs between the links to find the path with minimum average cost. The chosen path then becomes the most 'reliable' path.

The rest of the paper is organized as follows. Section 2 gives an overview of our protocol including a discussion on cost function in subsection 2.1. We introduce our trust metric in section 3 where the components and the methods to compute them have been discussed in subsections 3.1, 3.2 and 3.3 followed by the computation of the final trust value in subsection 3.4 . Subsequent section 4 presents our trust-based routing protocol. In section 5 we analyze our protocol. We present the security analysis of the protocol in subsection 5.1. In subsection 5.2 we discuss the complexity of our protocol. We start with computation complexity followed by communication complexity and storage complexity respectively. Finally, we conclude our discussion in section 6 with a summary for future work.

\section{Overview of Trust-Based Routing Protocol}

We assume that the pervasive computing environment supporting the application has a very dynamic topology. Nodes join or depart the environment at random. Each node in the network maintains a table $R T$ consisting of tuples of the form $\langle$ Dest, Win\#, NH, Cost $_{\text {avg }}, \sum$ Cost $\left.^{2}, \# H o p s\right\rangle$. In this table the node stores on a per-destination (Dest) basis, the identity of the next neighbor $(N H)$, to which the message needs to be forwarded. Together with the next neighbor information, the node also stores the minimum average cost $\left(\right.$ Cost $\left._{\text {avg }}\right)$ and the number of hops (\#Hops) to reach the particular destination. This information is generated periodically. Thus each tuple bears a time stamp in the form of a current time window (Win\#). The routing algorithm that we propose is used to update the next neighbor entry in the $R T$ table for a particular destination.

A source node initiates the routing protocol if it has a packet to be sent to a destination for which it does not have any next hop $(\mathrm{NH})$ entry. The source node can also execute the routing protocol when the path to the destination has expired (when the value under Win\# is less than the current window number). The source sends out a route 
discovery request. We assume that each node that participates in the pervasive computing environment has a trust relationship with its neighbor (that is a node at 1 hop distance). A trust-aware node periodically sends a beacon message to its neighbors. The beacon message is something like an "I am alive" message and carries information necessary to prove the node's existence. Once in a while a node can also send out a beacon message on demand. In our protocol, a node may request a recommendation score from a second node about a neighbor of the second node. In such cases the recommendation score is carried on a beacon message. Beacon messages are broadcast in nature. They carry rudimentary checksums to provide weak protection against integrity violations. The recommendation score is used as one of the parameters for computing trustworthiness of a neighbor. Trust relationships are periodically refreshed locally. At some point after system initiation we assume that every node in the system will have a trust relationship with each of its neighboring nodes. We do not assume that trust relationships are symmetric or transitive.

We adapt the trust model proposed in [15]. We express the trust relationships between nodes as $N_{r} \longrightarrow N_{e}$ where $N_{r}$ is the truster node and $N_{e}$ is the trustee node. We represent this trust relationship as a tuple $\left({ }_{N_{r}} P_{N_{e}}, N_{r} R_{N_{e}},{N_{r}}_{r} I_{N_{e}}\right)$. The value ${ }_{N_{r}} P_{N_{e}}$ represents an evaluation of the physical properties of $N_{e}$ by $N_{r}$. The value ${ }_{N_{r}} R_{N_{e}}$ denotes an evaluation of the recommendation scores of $N_{e}$ from other nodes and ${ }_{N_{r}} I_{N_{e}}$ evaluates the interactions that $N_{r}$ had with $N_{e}$. The exact interpretation of these terms are deferred for the time being till the next section (section 3). We associate a numeric value $v\left(N_{r} \longrightarrow N_{e}\right)$ (from $[-1,1]$ ) with the above tuple which we refer to as the trust value for node $N_{r}$ on node $N_{e}$ along the edge $\left(N_{r}, N_{e}\right)$. We next convert this trust value to a cost on the link $\left(N_{r}, N_{e}\right)$. The higher the trust value the lesser is the cost to transfer messages on the link. The path having the least average cost from the source node to the destination node is considered the most reliable among the available paths and is chosen by the source node to forward the data.

Figure 2(a) describes pictorially the main idea of our protocol. We assume that if a node $N_{r}$ has a distrust value (that is value less than 0 ) on another node $N_{e}$ the cost on that link is infinite and that next hop is discarded. When a node receives a route discovery request from a source, it checks its routing table $R T$. If a route to the destination is present in $R T$ which has not expired, it sends the '\#Hops' and cost related information

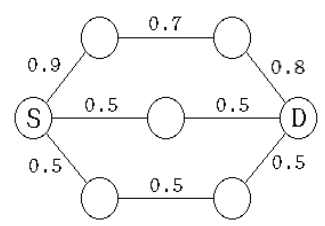

(a) The trust relations in trust-aware pervasive computing environment

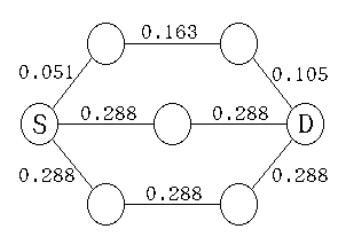

(b) The forwarding cost in trust-aware pervasive computing environment

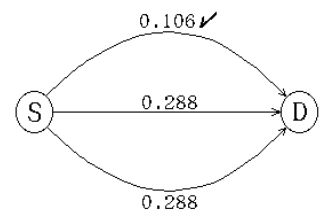

(c) Path having least average cost is most reliable

Fig. 2. Trust relation between nodes and the corresponding cost on the link 
to the source. The source then evaluates the cost of the link between the neighbor and itself and using the \#Hops it computes the average cost of forwarding the packet to the destination. The source may get multiple such responses. It then chooses the next hop for which the average cost over the path is minimum. If the node that receives a route discovery request from the source, does not itself have the next hop information in its $R T$ for that destination, it initiates a route discovery process as a source. This process can go on till the node which is 1 hop behind the destination initiates a route discovery request.

\subsection{Cost Function}

Each node tries to find the path to a given destination which has the minimum average forwarding cost. The cost of forwarding a packet from the node $N_{r}$ to $N_{e}$ is a function of $v\left(N_{r} \longrightarrow N_{e}\right)$. These two are related as follows: higher the trust, lesser is the cost and the cost increases as the distrust increases (i.e., the trust decreases). Rationale is, the cost (in terms of integrity violation and other malicious activities) of forwarding a packet through a more trustworthy node is less than that through a less trustworthy node. The cost is minimum (not zero though) when $N_{r}$ has absolute trust $\left(v\left(N_{r} \longrightarrow N_{e}\right)=1\right)$ on $N_{e}$. This minimum cost $\left(\right.$ Min $\left._{\text {cost }}\right)$ is a small positive cost incurred due to forwarding overhead. It is uniform over the whole pervasive computing environment and set at the bootstrapping of the system. We assume that the decay in cost with increased trustworthiness is logarithmic with the following conditions: at $v\left(N_{r} \longrightarrow N_{e}\right)=1$, cost $=$ Min $_{\text {cost }}$ and at $v\left(N_{r} \longrightarrow N_{e}\right)=-1$, cost $=\infty$. The function is defined as,

$$
\operatorname{cost}\left(N_{r}, N_{e}\right)=\operatorname{Min}_{\text {cost }}-\ln \left(\frac{1+v\left(N_{r} \longrightarrow N_{e}\right)}{2}\right)
$$

The maximum allowable cost for $N_{r}$ is incurred when $v\left(N_{r} \longrightarrow N_{e}\right)=0$. This corresponds to the situation when $N_{r}$ is neutral about trustworthiness of $N_{e}$. This cost, denoted by MaxAllowed $\operatorname{Most}_{\left(N_{r}, N_{e}\right)}$, is $\operatorname{Min}_{\text {cost }}-\ln \left(\frac{1}{2}\right)$, that is, $\operatorname{MaxAllowed}_{\operatorname{cost}\left(N_{r}, N_{e}\right)}=$ $\operatorname{Min}_{\text {cost }}+0.69$.

Computing this cost value has some overhead but is only linear in the number of nodes in the pervasive computing environment. The cost value is stored in the mobile device for a predetermined time or until a new beacon message has arrived. The absence of a beacon message from a particular node in a particular window of time represents a broken link during that time period. It can happen for various reasons including that the node is compromised. The node in such a case may either discard the broken link from the list of current neighbors or mark it as unused. Routing information is advertised by broadcasting the route setup packets periodically or on demand depending on the protocol used. These packets indicate which mobile nodes are accessible from which others and the average cost associated with the path towards a destination. When a node receives a data packet, it chooses the path which has the lowest average forwarding cost and forwards the packet to the neighbor on this path to be further forwarded towards the destination. During this process, a node also evaluates the packet forwarding performance of the neighbor node. By measuring this the node essentially evaluates an interaction score for the neighbor. The details of this process and other routing processes is explained in section 4 and section 5.2 


\section{Trust Metric}

As mentioned earlier in section 2 the trust of $N_{r}$ on $N_{e}$ depends on three factors $-N_{e}$ 's properties, recommendation about $N_{e}$ (alternatively, $N_{e}$ 's rating) from another node $N_{k}$, and $N_{r}$ 's interaction with $N_{e}$. We assume that each of these three factors is expressed in terms of a numeric value in the range $[-1,1]$. A negative value for the component is used to indicate the trust-negative type for the component, whereas a positive value for the component is used to indicate the trust-positive type of the component. A 0 (zero) value for the component indicates trust-neutral. The final trust value, denoted by $v\left(N_{r} \longrightarrow N_{e}\right)$, is calculated as an average of these component values. Eventually $v\left(N_{r} \longrightarrow N_{e}\right)$ falls in the range $[-1,1]$. A trustee node is completely trusted (or distrusted) if the value of the trust relationship is $1(-1)$. If the value is in the range $(0,1)$ the node is semi-trustworthy; if the value is in the range $(-1,0)$ the node is semiuntrustworthy. The 0 value represents trust neutrality, that is the trustee is equally trustworthy as untrustworthy.

\subsection{Computing properties}

In our approach trust is used as a reliability metric of a neighbor node for proper handling and forwarding the packet to the destination. A node $N_{i}$ is a neighbor of node $N_{j}$ if $N_{i}$ is within the range of a beacon message from $N_{j}$. A node becomes more reliable when it has relatively more resources (in terms of signal strength, signal stability, less propensity to corrupt data etc.). Higher values of these attributes show that it is more capable of handling and forwarding a packet in a reliable manner. This motivates us to measure the node properties quantitatively and include that measure as a factor to evaluate trustworthiness of a node. We focus on two properties of a node - signal strength, and stability factor. A node maintains a property table, $P T=\left\langle\right.$ Node_id, $\left.S S_{a v g}, S F\right\rangle$ for each neighbor node where the properties of the neighbor is kept along with the corresponding id. The table is updated after each time-window win.

Measuring signal strength. In each time-window win, a node periodically sends a link layer beacon message to its neighbors. When the neighbor node receives such a beacon message, the extended device driver interface of the receiving node measures the signal strength at which the beacon was received. In our approach we use the receive signal strength indicator (RSSI) unit to measure the signal strength. RSSI is the IEEE 802.11 standard for measuring radio frequency (RF) energy sent by the circuitry on a wireless network interface card (W-NIC). It is a numeric integer value with an allowable range of 0 to 255 . However, for the sake of our model we give a transformation to this recorded signal strength value by dividing it by 255 . This scales the received signal strength value within the range $[0,1]$. We require this transformation as the final value of the component 'properties' lies within $[-1,1]$. At the end of each time-window, we take the average of these values. This transformed average signal strength value is then stored under the column $S S_{\text {avg }}$ in the table $P T$ corresponding to the neighbor. All these signal strength values within the time-window win is kept in a separate temporary property table, $P T_{\text {tmp }}^{\text {Node_id }}=\langle S S, S B\rangle$ where $S B$ is the stability bit explained next. 
Measuring stability factor. The stability factor indicates the stability of a node. Higher the stability more reliable is the node to forward a packet. We derive the stability factor using signal strength. The reason is as follows: if a node is locationally unstable, it will have a varying signal strength. Alternatively, if the average signal strength of a node is fairly constant over few time-windows, the link with the node can be considered as stable. Therefore, after storing the strength of received signal, say $S S_{\text {current }}$, in $P T_{t m p}^{\text {Node_id }}$, it is compared with the value present in $S S_{a v g}$ of $P T$. If $S S_{\text {current }}<S S_{\text {avg }}$ then the $S B$ is set to 0 , otherwise the default value 1 is kept. At the end of time-window, the stability factor $S F$ is calculated as,

$$
S F=\frac{\text { number of bits set to } 1 \text { under } \mathrm{SB}}{\text { Total number of bits under } \mathrm{SB}}
$$

At the beginning of each time window the temporary property table $P T_{t m p}^{N o d e}{ }_{-} i d$ is set to its default values. The default value for $S S$ is 0 and for $S B$ is 1 .

Measuring properties. The properties component of the node $N_{e}$ is then computed as

$$
{ }_{N_{r}} P_{N_{e}}=\alpha * S S_{a v g}+(1-\alpha) * S F
$$

where $\alpha \in(0,1)$ is a fraction used as the relative importance weight to the signal strength property.

\subsection{Computing recommendation}

Each trust-aware node agrees to provide a 'recommendation' about its neighbors upon receiving a recommendation request from a source node. Let $N_{r}$ request $N_{k}$ for a recommendation about a node $N_{e}$. The source node $N_{r}$ sends this recommendation request by sending a special message REC_REQ containing the node_id of the target node (in this case $N_{e}$ ). The node $N_{r}$ can choose this recommender using a threshold_trust value $T_{t h r}$. That is, if $v\left(N_{r} \longrightarrow N_{k}\right) \geq T_{t h r}$ then only $N_{r}$ sends a REC_REQ message to $N_{k}$. If $N_{k}$ has a trust relationship with $N_{e}$, then $N_{k}$ replies by sending a message REC_RESPONSE containing the pair $\langle$ node_id, $V\rangle$ where $V=v\left(N_{k} \longrightarrow N_{e}\right)$. The node $N_{r}$ then scales this recommendation with the trust value that it has on $N_{k}$. Averaging all such recommendation received gives the 'recommendation' (or, rating) of $N_{e}$. Formally, if $N_{r}$ receives $m$ recommendations about $N_{e}$, then

$$
N_{r} R_{N_{e}}=\frac{1}{m} \sum_{k=1}^{m}\left\{v\left(N_{r} \longrightarrow N_{k}\right) \times v\left(N_{k} \longrightarrow N_{e}\right)\right\}
$$

The truster $N_{r}$ maintains a list, called recommendation list, $R L=($ node_id,list $)$ for each trustee where structure of each item in the list is (node_id, recommendation_value).

\subsection{Computing interaction}

Interaction is modeled as cumulative effect of events encountered by a truster node $N_{r}$ regarding $N_{e}$. We classify interaction in two categories - packet forwarding interaction - when the truster considers the behavior of the trustee as a packet forwarder, and rating 
interaction - when the truster considers the behavior of the trustee as a recommender. Every event in each of these categories has binary outcome; either the truster $N_{r}$ has trust-positive event or a trust-negative event depending whether the event contributes toward a trust-positive interaction or a trust-negative interaction.

Evaluating packet forwarding interaction. To evaluate packet forwarding interaction, the truster $N_{r}$ checks the outcome of each packet forwarded to $N_{e}$ within the specific time window win. Each packet forwarded correctly towards the destination is considered as a trust-positive event. Each dropped packet gives rise to a trust-negative event. The node $N_{r}$ measures the number of forwarded packet by $N_{e}$ as follows: $N_{r}$ forwards packets to $N_{e}$ and with every such packet $N_{r}$ sends an ECHO message with a time-to-live $(\mathrm{TTL})=2$. Each reply received by $N_{r}$ denotes correct forwarding of the packet by $N_{e}$ to the next member $N_{k}$ in the path. $N_{r}$ keeps this information in a table $I T=\left\langle\right.$ Node $\left._{-} i d, P F C_{p}, P F C_{n}, R C_{p}, R C_{n}\right\rangle$ where $P F C_{p}$ denotes the counter for trust-positive packet forwarding interaction within the window and $P F C_{n}$ counts the trust-negative packet forwarding interactions. $R C_{p}$ and $R C_{n}$ are rating counters used for counting the results of rating interactions. All these fields has default value 0 . Whenever a packet is dropped the counter $P F C_{n}$ is increased by 1 and for each received reply the counter $P F C_{p}$ is increased. Formally, packet forwarding interaction, denoted by $I_{p f}$ of $N_{r}$ about $N_{e}$ within the window win is defined as the ratio $\frac{P F C_{p}-P F C_{n}}{P F C_{p}+P F C_{n}}$.

Evaluating rating interaction. The rating interaction is evaluated in a similar manner. We assume that each node agrees to provide a 'trust recommendation' about its neighbors upon receiving a recommendation request from a source node. We also assume that for each neighbor, the truster keeps a list of node_ids of the nodes who have provided recommendation for that neighbor. Whenever the truster has a 'packet forwarding interaction' with the neighbor node and the result of that interaction matches with the recommendation, that is the truster has positive (negative) experience and the recommendation is also positive (negative), it increases the $R C_{p}$ in $I T$ of all such recommenders by 1 . If there is a mismatch between the outcome and the recommendation, the truster increases the $R C_{n}$ counter by 1 for those recommenders. For example, let the trustee $N_{e}$ has provided "positive" recommendations for the nodes $N_{i}, N_{j}, N_{k}$ to the truster $N_{r}$. Therefore, in the recommender list $R L, N_{e}$ appears in the list against each of these nodes. Let $N_{r}$ have trust-positive packet forwarding interaction with $N_{i}, N_{j}$ and trust-negative packet forwarding interaction $N_{k}$. Then in the interaction table $I T$, for the node $N_{e}$, the counter $R C_{p}$ is increased twice and $R C_{n}$ once. At the end of time window win the rating interaction, denoted by $I_{r}$ of $N_{r}$ about $N_{e}$ is defined as the ratio $\frac{R C_{p}-R C_{n}}{R C_{p}+R C_{n}}$.

Evaluating interaction. The interaction component of the node $N_{e}$ is evaluated as

$$
{ }_{N_{r}} I_{N_{e}}=\beta * I_{p f}+(1-\beta) * I_{r}
$$

where $\beta \in(0,1)$ is a fraction used as the relative importance weight to the packet forwarding interaction. 


\subsection{Computation of Final Trust Value}

After computing values of the components we evaluate the trust value for the trustee $N_{e}$ as the average of the components. Formally,

$$
v\left(N_{r} \longrightarrow N_{e}\right)=\frac{N_{r} P_{N_{e}}+{ }_{N_{r}} R_{N_{e}}+{ }_{N_{r}} I_{N_{e}}}{3}
$$

These information are kept in a trust table, $T T=\langle$ node_id, properties, recommendation, interaction, trust_value, cost $\rangle$. After each window win this table is updated with new values which are kept and used in the next time window. All other tables are set to their corresponding default values. Next section describes the modified distance vector routing algorithm which finds the path with minimum average cost for forwarding a packet to the destination.

\section{Data Path Discovery}

To select the most trustworthy path, each node evaluates and dynamically updates the trust components between itself and current neighbors. It then calculates the trust value of the neighbors by the process described in section 3 . These values are used to calculate the forwarding cost between two neighbors, using the equation 1 in section 2.1 The path with the minimum average forwarding cost is preferred and the adjacent node on this path is trusted to forward the packets toward the destination.

\subsection{Route Discovery}

Our algorithm is based on a "rumor" about paths from neighbors. This is incomplete information. We thus choose to use the average and standard deviation of the running sum of cost in our route discovery protocol. This formula does not require the complete path information yet can correctly evaluate the path's reliability like the one with the complete path information. The average and standard deviation of running sum are computed as follows: let, a random variable $X$ take on the values $x_{1}, \ldots, x_{n}$ and $x$ be the latest value. We use the following equations:

$$
\begin{gathered}
A V G=\left(x+\sum_{i=1}^{n} x_{i}\right) /(n+1) \\
S D=\sqrt{n \sum_{i=1}^{n} x_{i}^{2}-\left(\sum_{i=1}^{n} x_{i}\right)^{2} / n(n-1)}
\end{gathered}
$$

When a node receives a route information message from a neighbor node $N_{k}$, it updates the forwarding cost on the path towards node $N_{j}$ (where node $N_{k}$ is chosen as the next hop) by adding the current cost between itself and $N_{k}$ and calculate the updated average cost using equation 6 It then re-evaluates the path to choose the optimal route to the destination. This process compares among all possible candidate routes and chooses the path that has the minimal average cost. If more than one candidate paths have same minimum average cost or have a difference of cost less than a given threshold $\eta$, the routing algorithm selects the path that has the least standard 


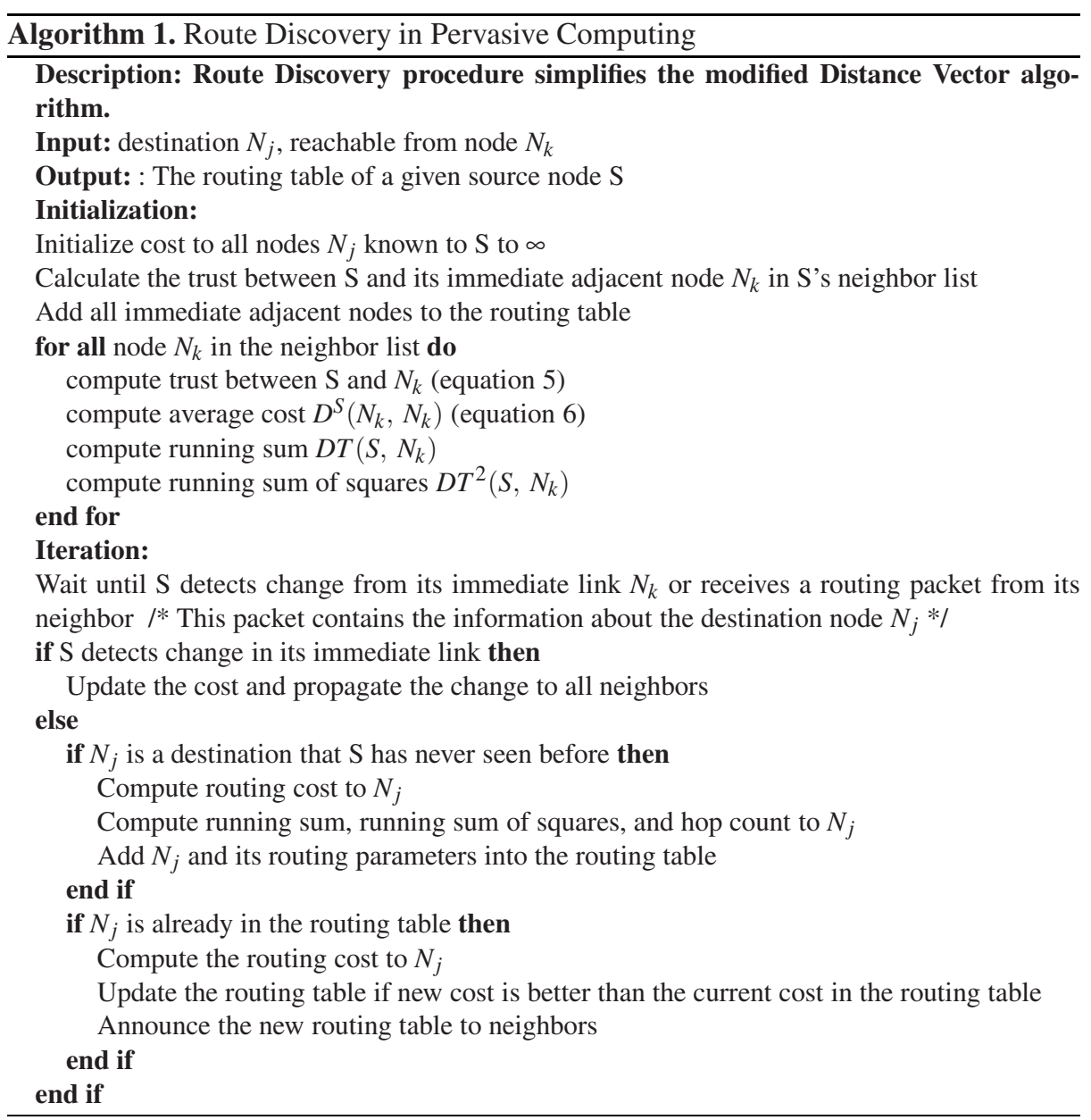

deviation as an optimal path. The standard deviation is calculated using the equation 7 . Algorithm 1 gives the protocol used in generating the routing table. It consists of two phases: table initialization and iteration. The table initialization phase establishes paths to all immediate neighbors known to the source S. For each neighbor $N_{k}$, node $\mathrm{S}$ keeps track of hop count, average cost (calculated from equation [5], running sum of cost $\left(D T\left(S, N_{k}\right)\right)$, and running sum of square of cost $\left(D T^{2}\left(S, N_{k}\right)\right)$. These cost parameters are used for calculating the average cost and standard deviation according to the equation 6 and equation 7 The iteration phase is only triggered upon receiving the routing packets or upon changing of an immediate link with its neighbor.

In the first case, if the destination node $N_{j}$ in the received packet is not known by node $\mathrm{S}$, it will add $N_{j}$ to the routing table and compute the routing cost to $N_{j}$ by adding its trust between itself and its neighbor node who has sent the routing information of $N_{j}$ to $\mathrm{S}$. The routing cost to the destination $N_{j}$ is computed as: 


$$
D^{S}\left(N_{j}, N_{k}\right)=\frac{v\left(S \longrightarrow N_{k}\right)+D T\left(N_{k}, N_{j}\right)}{\text { hop_cnt }\left(N_{k}, N_{j}\right)+1}
$$

where Node $N_{k}$ is the sender of the routing information and $D T\left(N_{k}, N_{j}\right)$ is the forwarding cost from $N_{k}$ to $N_{j}$. If $\mathrm{S}$ already knows the destination node $N_{j}$, it recomputes the routing cost to $N_{j}$ and compares this value with the existing value. If the new cost is less or more stable 1 than the current cost, the cost to the destination $N_{j}$ is updated. If the trust value between node $\mathrm{S}$ and its immediate neighbor $N_{k}$ has changed, $\mathrm{S}$ has to recompute the routing cost to all destinations $N_{j}$ where $N_{k}$ is the next hop. The above equation 8 is used to recomputing the new routing cost. Then $S$ compares the new cost to the current cost that $\mathrm{S}$ has in its routing table. If the new cost is less or more stable than the current cost, the cost to the destination $N_{j}$ is updated.

\section{Analysis}

\subsection{Security Analysis}

The trust-based approach to routing is intended to minimize the effect of malicious nodes in the network. We discuss how the proposed scheme can reduce this effect. A malicious node can subvert the network in two ways:

Dropping packets. A malicious node on a path can deliberately drop the legitimate packets. Suppose a node $N_{i}$ is sending a packet to $N_{j}$ through the neighbor $N_{m}$ who is malicious and drops packets arbitrarily. With every drop of packet, $N_{i}$ increases the 'trust-negative packet forwarding counter' $P F C_{n}$ corresponding to the node $N_{m}$ in the interaction table. Note, in our scheme $N_{i}$ cannot differentiate between a deliberate drop of packet and a packet drop due to valid reasons (like broken link or downtime of a node). However for a malicious node, number of dropped packets will be high compare to number of forwarded packets. This will lower the ratio $\frac{P F C_{p}-P F C_{n}}{P F C_{p}+P F C_{n}}$ and consequently $N_{i}$ 's trust on $N_{m}$ will be low. Even if $N_{m}$ keeps oscillating packet drop behavior, the above ratio will be close to zero and does not help $N_{m}$ to increase its trust. Also note that $N_{m}$ cannot fool $N_{i}$ by dropping the actual data packet but forwarding the ECHO message to $N_{j}$. Because $N_{j}$ will not reply to the ECHO message unless it receives the corresponding data packet.

Providing false recommendation. A malicious node $N_{m}$ can disrupt the proper functioning of the scheme by providing false recommendation about a node. Suppose the malicious node $N_{m}$ provides a "positive" rating about nodes $N_{k}, N_{l}$ where both of them are malicious nodes. Every time $N_{i}$ encounters a trust-negative packet forwarding event with any of them, $N_{i}$ increases the 'trust-negative rating counter' $R C_{n}$ of $N_{m}$ which lowers the ratio $\frac{R C_{p}-R C_{n}}{R C_{p}+R C_{n}}$. Therefore, even if $N_{m}$ behaves properly in the context of packet forwarding, it cannot subvert the system by falsely 'campaigning' for some other malicious nodes in the network. This also reduces the effect of collusion of malicious nodes

\footnotetext{
${ }^{1}$ This is used in the case when the new cost is equal to the the current cost or has a slight difference. The path that has less standard deviation is said to be more stable path.
} 
to disrupt reliable routing. Similar action prevents the problem of 'badmouthing' i.e., when $N_{m}$ provides false 'negative' rating about a 'good' node. This is possible because the trust of the benign node is not dependent just on rating provided by $N_{m}$, but involves other parameters on which $N_{m}$ cannot have any control.

The above discussions show that the proposed trust-based routing scheme can reduce the effect of malicious nodes - working as individual or as a part of collusion, in attacks like arbitrary packet drops, false data injection and badmouthing.

\subsection{Complexity Analysis}

As mentioned earlier, a typical device in a pervasive computing environment has relatively low resources in terms of storage and power. However, it needs to do some computations to evaluate the trust and cost. It also needs to store some values for a specific duration. In this section we analyze the computation, communication, and storage complexity of our protocol.

Computation complexity. In our approach the run-time complexity of the routing algorithm is not affected to a great extent. Our scheme only changes the metric of computing the administrative distance between nodes in the pervasive computing environment. It requires two additional computations for the running sum and the standard deviation. These additional computations do not change the big-O complexity of running time of the routing protocol as they are proportional to the size of the network. However, our approach requires additional methods in order to evaluate the trust between nodes. All these computations are simple arithmetic computations and have linear bounds. Consequently these do not add much to the computation overhead of the proposed protocol.

Communication complexity. A node maintains view of connectivity and trust relationship by periodically transmitting a beacon packet. This packet carries the node information including sequence number, hardware address, hardware protocol, and trust recommendation upon request. According to this scheme, there are 3 types of the beacon message - announcement, recommendation request, and recommendation reply. When a node receives a beacon message, it identifies the sender, measures the strength of the beacon signal (section 3.1), collects the trust information and recalculates the trust value of the sender. If the beacon message is a recommendation reply and the trustee is recognized by the receiver, it recomputes the trust value of the trustee with this recommendation information about the trustee. If the beacon message is a recommendation request message, and the trustee is recognized by the receiver, it prepares the trust value of the trustee and sends it to the requester with the next beacon message. We have estimated that a message 256 bits (32 bytes) long is sufficient to carry the beacon message. In the following discussion we show that routing tables in our protocol require only 154 bits for each record. Therefore, for a small network (say, with 50 nodes), the nodes pass a routing table which is of size less than $1 \mathrm{~KB}$.

Storage complexity. In our protocol each node has to maintain a certain number of tables. In this section we discuss the storage overhead that is required to store these 
tables. For each neighbor, a truster node needs to maintain the following tables and list: $P T$ (properties table), $P T_{t m p}^{\text {node }}$ id (temporary property table), IT (interaction table), TT (trust table), and the list $R L$. In any table the node_id field takes 32 bits to store the address. We express the values of $S S_{a v g}$ in $P T, S S$ in $P T_{t m p}^{\text {node }}$ id , and Properties, Recommendation, Interactions, Trust in TT using 16 bits in which the most significant bit is the sign bit, the next bit expresses the exponent, and the rest 14 bits expresses the fraction. We need only 1 bit to express the exponent as all these numbers are within $[-1,1]$. Also, we get the precision of $1 / 2^{14}$ for trust related values. In the interaction table $I T$ we use 8 bits to express each counter. The signaling factor is also expressed using 8 bits. However, we use 32 bits to represent the cost in TT as it is not bounded. Since the cost is always positive, we use first 16 bits for the exponent and the last 16 bits for the fraction. This gives the precision of $1 / 2^{16}$ for the cost value which is accurate enough for the environment. The figure 3(a) shows the structure of the tables stored for each neighbor. From the figure we see that the tables $P T, I T, T T$ require 56,64 and 128 bits respectively for each record. Each of these tables is maintained for all the neighbors. If a node has $m$ neighbors, then it requires $m \times 228$ bits. To store each signal and stability information we need 24 bits. If we assume that a node receives $s$ signals within a window, then for each neighbor it needs $32+s \times 24$ bits to store the signal. Hence for all $m$ neighbors the node requires $m .(32+s .24)$ bits. For the recommendation list $R L$ each record requires 48 bits and we assume at most $k(k \leq m)$ recommenders recommend a neighbor. Therefore for each neighbor it requires $32+k \times 48$ bits and hence for $m$ neighbors $m .(32+k .48)$ bits. Note, we have not considered the pointer size here as it depends on the implementation. Hence for each neighbor a node requires $228+(32+24 . s)+(32+k .48)=292+24 . s+48 . k$ bits and for all $m$ neighbors

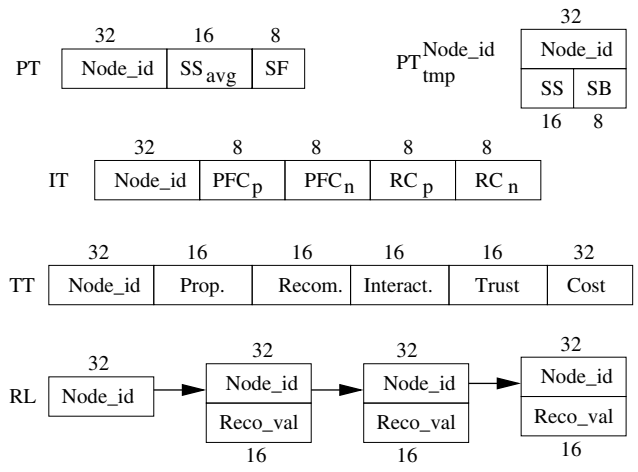

(a) Tables maintained for each neighbor

\begin{tabular}{cc|c|c|c|c|c|}
\multicolumn{2}{c}{32} & \multicolumn{1}{c}{8} & 32 & 32 & \multicolumn{1}{c}{8} & 32 \\
\cline { 2 - 7 } RT Destination & Win\# & NH_id & Cost $_{\text {avg }}$ & \#Hops & Sum $^{2}$ \\
\cline { 2 - 6 }
\end{tabular}

(b) Routing table of the node

Fig. 3. Storage structure of the tables maintained in each node 
total storage required is $m .(292+24 . s+48 . k)$ bits. This storage is not significant if we assume that each node in the pervasive computing environment interacts with a small number of neighbors. For example, in a pervasive computing environment topology where each node interacts with at most 20 neighbors and at most 100 signals are received within a time window win, then maximum number of bits required to store the trust information is $(292+24 \times 100+48 \times 20) \times 20=3652 \times 20=73040$ bits $\approx 9 \mathrm{~KB}$.

Each node also stores a routing table whose structure is shown in the figure $3(\mathrm{~b})$ The Win\# field keeps the last window number. \#Hops stores the number of hops to the destination. NH_id field stores the address of the next hop towards destination. Cost $_{a v g}$ and $\Sigma^{2}$ fields store the cost metrics which are used in the route selection protocol. We also need the metric $\sum$ for route selection. However we do not store this information in

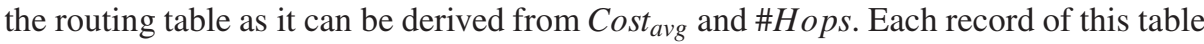
requires 154 bits and hence size of the routing table for each node in the topology is $O(154 n)$ where $n$ is the number of nodes in the network. In our example if we assume a small network with 50 nodes then each node require maximum $154 \times 50$ bits which is $\approx 0.9 \mathrm{~KB}$. Therefore all together a node requires only $10 \mathrm{~KB}$ storage space to store the information related to trust-based routing.

The above analyses show that our protocol is light-weight in terms of trust evaluation, feedback management, message passing, and storage, thereby making it suitable for pervasive computing environment.

\section{Conclusion and Future Work}

In this work, we address the problem of reliable delivery of event data in pervasive computing environments to appropriate action points in order to support obligation policies. The problem is modeled as a routing problem. We present a trust-based approach to routing. Each node measures trustworthiness of its neighbor based on the neighbor's properties like signal strength and signal stability, a neighbor's behavior in forwarding packets from the node as well as in recommending other nodes, and a neighbor's rating by other neighbors. We represent each link in the network as a trust relationship with a numeric value between $[-1,1]$. This trust metric reflects reliability of a node as a packet forwarder. We have proposed a cost metric, which is inversely related to the trust metric, for each link in the network. We next adapt the distance vector routing algorithm for routing in pervasive computing environments. The modified algorithm uses the cost value assigned to each link to find the minimum average cost path from source to destination. We have discussed how our protocol can reduce the effect of malicious nodes. We have also shown that the scheme does not enhance the computation, communication, and storage overhead to any significant extent.

A lot of work still remains to be done. The proposed scheme is generic in nature. We need to modify specific ad hoc routing protocols using our metrics and compare the results to evaluate the performance of the proposed scheme. We plan to run simulation experiments to compare the routing results with existing ad hoc routing protocols. We are also looking for other node properties like remaining battery power to extend the 'properties' parameter of the trust metric. 


\section{References}

1. Balfanz, D., Smetters, D., Stewart, P., Wong, H.: Talking to Strangers: Authentication in Adhoc Wireless Networks. In: Symposium on Network and Distributed Systems Security (NDSS '02), San Diego, California, USA (February 2002)

2. Chiang, C.C., Wu, H.K., Liu, W., Gerla, M.: Routing in Clustered Multihop, Mobile Wireless Networks with Fading Channel. In: 5th IEEE Singapore International Conference on Networks (SICON'97), Kent Ridge, Singapore pp. 197-211 (April 1997)

3. Corson, M.S., Ephremides, A.: A Distributed Routing Algorithm for Mobile Wireless Networks. Wireless Networks 1(1), 61-82 (1995)

4. Gafni, E., Bertsekas, D.: Distributed Algorithms for Geneerating Loop-free Routes in Network with Frequently Changing Topology. IEEE Transaction and Communication 29(1), 1115 (1981)

5. Gerla, M., Tsai, J.T.: Multicluster, Mobile, Multimedia Radio Network. Wireless Networks 1(3), 255-265 (1995)

6. Hu, Y.C., Perrig, A., Johnson, D.B.: Ariadne: A Secure On-Demand Routing Protocol for Ad Hoc Networks. In: Proceedings of the 8th Annual International Conference on Mobile Computing and Networking (MobiCom'02), Atlanta, Georgia, USA (September 2002)

7. Papadimitratos, P., Haas, Z.: Secure Data Transmission in Mobile Ad Hoc Networks. In: ACM Workshop on Wireless Security (WiSe'03), San Diego, California, USA (September 2003)

8. Perkins, C.E., Bhagwat, P.: Highly Dynamic Destination-Sequenced Distance Vector (DSDV) for Mobile Computers. In: Conference on Communication Architectures, Protocols and Applications (SIGCOMM'94), London, UK, pp. 234-244 (August 1994)

9. Toh, C.K.: A Novel Distributed Routing Protocol To Support Ad hoc Mobile Computing. In: IEEE 15th Annual International Phoenix Conference on Computers and Communication (IPCCC'96), Phoenix, AZ, USA, pp. 480-486 (1996)

10. Zhou, L., Haas, Z.J.: Securing Ad Hoc Networks. IEEE Network 13(6), 24-30 (1999)

11. Zouridaki, C., Mark, B.L., Hejmo, M., Thomas, R.K.: A Quantitative Trust Establishment Framework for Reliable Data Packet Delivery in MANETs. In: Proceedings of the 3rd ACM Workshop on Security of Ad Hoc and Sensor Networks (SASN'05), Alexandria, VA, USA, pp. 1-10. ACM Press, NewYork (2005)

12. Awerbuch, B., Holmer, D., Nita-Rotaru, C., Rubens, H.: An On-Demand Secure Routing Protocol Resilient to Byzantine Failures. In: ACM Workshop on Wireless Security (WiSe'02), Atlanta, GA, USA, pp. 21-30 (September 2002)

13. Dube, R., Rais, C.D., Wang, K.Y., Tripathi, S.K.: Signal Stability-Based Adaptive Routing (SSA) for Ad Hoc Mobile Networks. IEEE Personal Communications Magazine 4(1), 36-45 (1997)

14. Yi, S., Naldurg, P., Kravets, R.: Security-Aware Ad Hoc Routing for Wireless Networks. In: Proceedings of the 2nd ACM Symposium on Mobile Ad Hoc Networking and Computing (MobiHOC 2001), Long Beach, CA, October 2001, pp. 299-302. ACM Press, New York (2001)

15. Ray, I., Chakraborty, S.: A Vector Model of Trust for Developing Trustworthy Systems. In: Samarati, P., Ryan, P.Y. A., Gollmann, D., Molva, R. (eds.) ESORICS 2004. LNCS, vol. 3193, pp. 260-275. Springer, Heidelberg (2004) 